\title{
summary
}

\section{No difference in quality of life between rigid and non-rigid fixation used in BOSS osteotomy}

Hatch et al. Health-related quality of life following orthognathic surgery. Int J Adult Orthod Orthognath Surg; 13:67-77

Objective To compare the effects of rigid and wire fixation on health-related quality of life following surgical mandibular advancement in patients with Class II malocclusions.

Design A multi-site randomised controlled trial.

Intervention One hundred and forty patients aged at least 13 (referred for surgical correction of a class II dentofacial malocclusion by orthodontists) were randomised to receive wire $(n=63)$ or rigid $(n=64)$ fixation. Strict exclusion criteria were used. Age, overjet, EPI Neuroticsm score, gender and ethnicity were analysed.

Outcome measures Quality of life was measured using the Sickness Impact Profile, a generic measure of health-related quality of life, and the Oral Health Status Questionnaire, a specific measure of oral health and function designed for use with orthognathic surgery patients. Patients were evaluated before application of orthodontic appliances, approximately 2 weeks before surgery; and 1 week, 8 weeks, 6 months, 1 year, and 2 years after surgery.
Results Neither the Sickness Impact profile or the Oral Health Status Questionnaire revealed a statistically significant difference in quality of life between wire and rigid fixation at any time period.

Conclusion The authors point out that the implicit assumption in orthognathic surgery, as in all surgery, is that patients accept short-term risks and discomforts in return for long-term benefits in terms of length or quality of their lives. They conclude the health-related disability associated with Class II malocclusion is modest compared with many other medical conditions. Nonetheless, orthognathic surgery patients exhibit progressive and statistically significant improvement in healthrelated quality of life across a wide variety of functional domains, regardless of the fixation method used. This study did not involve an untreated control group so the quality of life observed in the study subjects cannot be compared with that which might occur in untreated patients.

Address for reprints: Dr John R Hatch, Dept of Orthodontics, The University of Texas Health Science Center at San Antonio, 7703 Floyd Curl Drive, San Antonio, Texas 78284-7910, USA.

\section{Commentary}

The authors present an interesting study of the effects of orthognathic surgery for moderate class II skeletal malocclusions, treated using bilateral sagittal split osteotomies, on patientreported health-related quality of life. The health outcome measures are well chosen and include a generic measure (the sickness impact profile, SIP) and a specific oral health scale developed for the purpose of assessment following orthognathic surgery. They assess responses at seven time periods ending 2 years after surgery. This approach has the inevitable problem of repeated response bias, given that the patients respond to the same questions several times. Patients reported statistically significant quality of life improve- ments, using either outcome measure following surgery, although it is also notable that no patient scored sufficiently low on the SIP to be deemed to have a disease of clinical relevance before surgery. The authors also report there were no statistical differences in outcome at any time after surgery between cases treated using rigid fixation and those treated using non-rigid fixation. This is a surprising finding, given that there is wide consensus amongst the oral surgery community that rigid fixation offers significant lifestyle benefits for patients in the immediate period following surgery with earlier return to normal oral function. The study was based on a relatively small sample of North American subjects and the results should be applied with caution to European populations. The authors acknowledge the lack of control groups (including no treatment and orthodontic care alone). In addition, on the basis of the information reported, the cases include some that might be treated without surgery in the UK. Nevertheless, the study provides evidence that there is no difference in patient-perceived quality of life between rigid and non-rigid fixation. This deserves further investigation given the current strong trend towards abandoning non-rigid fixation in favour of rigid fixation.

\section{Mark Brickley}

Dept of Oral and Maxillofacial Surgery, Welsh National School of

Medicine, UK 